\title{
A Teledentistry System for the Second Opinion
}

\author{
Orazio Gambino ${ }^{1}$, Fausto Lima ${ }^{1}$, Roberto Pirrone ${ }^{1}$, Edoardo Ardizzone ${ }^{1}$, Giuseppina Campisi ${ }^{2}$ \& Olga di Fede ${ }^{2}$
}

\begin{abstract}
In this paper we present a Teledentistry system aimed to the Second Opinion task. It make use of a particular camera called intra-oral camera, also called dental camera, in order to perform the photo shooting and real-time video of the inner part of the mouth. The pictures acquired by the Operator with such a device are sent to the Oral Medicine Expert (OME) by means of a current File Transfer Protocol (FTP) service and the real-time video is channeled into a video streaming thanks to the VideoLan client/server (VLC) application. It is composed by a HTML5 web-pages generated by PHP and allows to perform the Second Opinion both when Operator and OME are logged and when one of them is offline.
\end{abstract}

\section{INTRODUCTION}

Nowdays, the best first step in every medical diagnostic process is the conventional oral examination (COE). Its effectiveness has been reported, in particular, for screening of oral potentially malignant ones [1] [2] performed by oral medicine doctors. In a territory where the number of oral medicine centers is scarce, the chance to provide a second qualified opinion formulated from the specialist centers to the generic dental practitioner becomes a crucial task for an accurate diagnosis. Moreover, often dental or oral medicine ward are not present inside the oncological centers, so these delicate patients don't receive medical assistance for the primary and secondary prevention of oral and dental diseases caused by the cancer treatments (i.e radiotherapy, chemotherapy, bisphosphonates, antiresorptive agents, antiangiogenic agents). For example, in Italy it is mandatory to perform the COE on the patient before starting a therapy based on the amino- bisphosphonates in order to be sure about their oral health [3] [4]. As a consequence, the use of Dental/oral Second Opinion Systems is rapidly growing up. Second Opinion systems can be considered a branch of Telemedicine: a mixture among Medicine, Telecommunication and Computer Science. Telemedicine systems are also developed for pervasive computing [5]. A sub-category of Telemedicine is the Teledentistry: here Medicine is substituted by the more specific Dentistry. Thanks to teledentistry, it is possible to perform distant consultations, sharing digital information like images,cooperative work, documents, radiographies and so on. The first project on Teledentistry dates from 1994: it was a military project of the United States Army (U.S.Armys Total Dental Access Project) [6]. Many other works oriented

\footnotetext{
${ }^{1}$ Dept. of Chemical Management Mechanical Engineering and Computer Science-University of Palermo,viale delle Scienze, Building 6 Email: orazio.gambino, lima.fausto, roberto.pirrone edoardo.ardizzone at unipa.it

${ }^{2}$ Department of Surgical, Oncological and Oral Sciences 'Giuseppe Messina'-University of Palermo, via del Vespro Email: giuseppina.campisi, olga.difede at unipa.it
}

978-1-4244-7929-0/14/\$26.00 (C2014 IEEE to Teledentistry can be found in literature. In [7] an IntraOral Camera (IOC) is used to acquire digital images of oral mucosal lesions. They are stored on a personal computer and transmitted via the Internet to a distant site and compared with the original ones. No loss of quality between transmitted and original images is noticed by a team of physicians. In [8] the occlusal lesions are better detected thanks to the use of the IOC.A Teledentistry system to increase and enhance the children's oral health quality in the rural regions of California is reported in [9]. In [10] a review on Teledentistry systems is reported. A Computer Supported Cooperative Work oriented to the cooperative oral medical diagnosis is described in [11]. A comparison between digital images of oral mucosal acquired with an IOC and a DSLR camera can be found in [12]. In [13] is described a Teledentistry system to perform remote diagnosis on children at school.The Operator captures the digital images using and IOC and transmits them to Oral Medicine Expert. In [14] another Teledentistry system for disadvantaged children is shown. In [15] is reported a distributed system for the oral health care information management. It allows to perform videoconference and sharing a variety of dental data. In [16] a Teledentistry system aimed to the diagnosis and routine management of endodontic/oral surgery patients is presented. A system devoted to the remote recognition of root canal orifices is presented in [17]; the images are captured by means of an IOC. In the present paper we show a system able to perform medical tele-reporting system, real-time video streaming and medical/radiological image sharing. The physician can supervise the Operator during medical examination thanks to the video streaming. Even if the Oral Medicine Expert is not logged into the system, both images captured with IOC and radiographic images can be transmitted by the Operator. A particular internet connection is not necessary: a common ADSL line or wi-fi connection can be used. The paper is organized as follows: Section 2 depicts the main hardware/software components of the system, Section 3 describes the role of the users, Section 4 a medical evaluation can be found and finally in Section 5 some conclusions and future works are reported.

\section{HARDWARE/SOFTWARE OVERVIEW}

\section{A. Hardware components}

A key device used in this project is the IOC. It is an optical device for dental use which allows the dentist to take photographs or make movies of the internal oral cavity and displays it on an external monitor. In the market there are different types of IOC with different features 


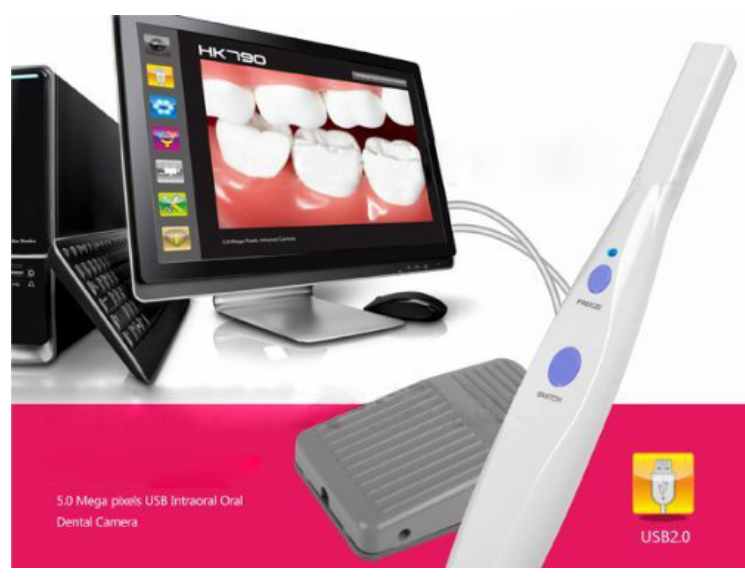

Fig. 1. The intra-oral camera HK 790.

and we have chosen the HK-790 (fig. 1). Such a device was chosen because it offers the best quality/price ratio. It is a 5.0 Megapixel CCD/CMOS device encapsulated in a package suitable to be introduced in the oral cavity, which is connected to the PC via USB 2.0 port, and the maximum resolution available is $2560(\mathrm{H}) \times 1920(\mathrm{~V})$. It has an antifog lens which prevents the formation of condensation and LEDs surrounding the lens for proper illumination of the oral cavity without the need for additional light sources.

\section{SOFTWARE COMPONENTS}

The system requires a web server to handle http requests and for our project Apache Web Server v2.2.8 has been chosen as web server. Furthermore, the applications MySQL v5.0.51b, for the management of persistent data, and phpMyAdmin, for the administration of the databases of the type DBMS (via a browser), have been chosen. These three applications were chosen because they are open source, cross-platform and these software solutions are most commonly used in their respective areas of competence.These applications are all grouped together in a software package known as AppServ 2.5.10. In addition, both the FTP Filezilla server as the application that handles files transfers and the VLC player (a software that is part of the VideoLAN project) as video streaming handler are used. The latter one is not only a multimedia player with many types of video codecs, but also it acts as a streaming server and client. Finally, the last component that completes the set of software used in the system is the application called USB Dental, which is provided by the manufacturer of the HK-790 IOC and allows to execute two functions: video grabbing and image acquisition.

\section{The Roles OF THE SyStem ACTORS}

The user can be logged according to one of three user types: Administrator, Operator, OME. The Administrator manages both the OME, Operator and other Administrator accounts 3-left. He/she gives or takes away permissions for registration to an OME. Each OME can be registered him/herself or an account can be assigned by the Administrator. The Operator is in direct contact with the patients and

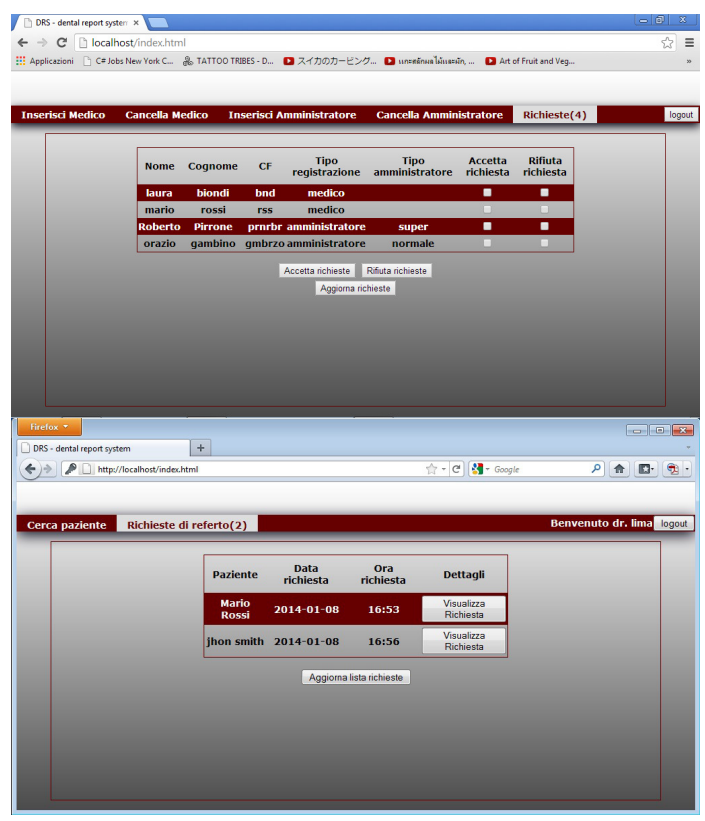

Fig. 3. From up-to-down: screenshot of the Administrator page for the accounts management; screenshot of medical report requests.

provides to the OME the data necessary to carry out the visit. In particular, the Operator can insert a new patient before to start a medical examination; he/she can send files to an OME, such as images and DICOM files related to the oral cavity of a patient; finally, he/she is able to send real-time video stream to an OME during the visit to a patient, only if the OME is logged to the account. During the real-time video streaming, the OME can view the patient's mouth directly through the IOC used by the Operator, who can receive instructions by the OME to perform new image acquisitions. A request of medical report may be sent to each OME by the Operator even though the OME is not logged to the account 3-right. The OME can create a new report by selecting a specific patient and accessing its own profile view images, DICOM files, and previous reports related the patient, giving a second opinion via a medical report. The OME can manage the medical reports; in particular, the system shows a page with a list of all the medical reports and from this page the OME can select the reports that he/she wants to delete, edit or to export in pdf format. All the relations among the actors is summarized in the Entity-Relationship Diagram in fig.3-up

\section{Medical Evaluation}

Mucosal lesions are very frequent in the oral cavity, they can be innocuous, chronic, but sometimes life threating, like when a potentially malignant lesion or a frank carcinoma is diagnosed. The diagnosis of oral mucosal lesions needs always a first preliminary step: the conventional oral examination (COE); thanks to this, in presence of an adequate source of white light, it is possible for an expert in oral medicine to start performing one of the following processes: a)pattern recognition (immediate recognition and definitive diagnosis of a given disease); b) algorithm, as logic sequence of signs and symptoms for suspicions more or 


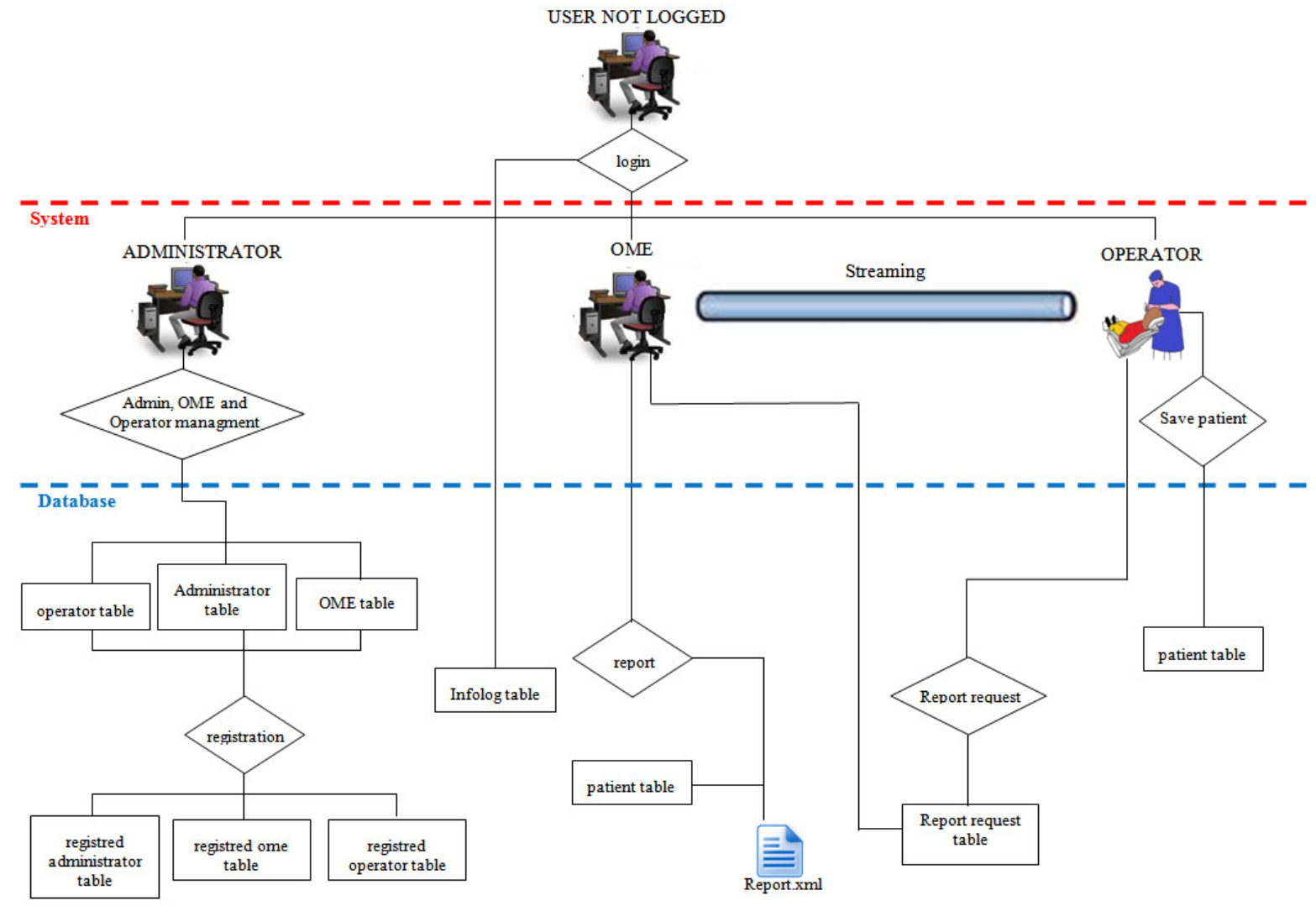

Fig. 2. The workflow of the system use.

less confirmed or ruled-out up to the most reliable clinical diagnosis; c) exclusion true (to reach a diagnosis as process of progressive exclusion of similar possible clinical forms); and d) hypothetic-deductive method, in which the expert knows the answer and works for establishing if it is correct. Any process we choose, we need always the COE; the present work can allow to work also in a remote mode (by acquisition of images or videos), with a potential massive medical impact on the community. The pillar of the oral clinical diagnosis is the ocular recognition of the variants from the normality (according to form and colour); these are classified in elementary lesions (e.g. macula, papula, plaque, vesicle, bulla, ulcer, neoplasia, tumefaction), but also in colors different from the normal pink one, the main are white, red, black, and blue. As example, we show some pictures acquired by our system and commented at distance in blindness.

Patient 1 of fig.4-top with clear reticular white lesion on the left cheek, strongly evocative of a case of lichenoid lesions. Patient 2 of fig.4-middle shows a red ulcerate area covered by yellow pseudo-membrane, with clean-cut margins, surrounded by white lesions suggesting a provisional diagnosis of immune muco-cutaneous disease.

Patient 3 of fig.4-bottom, site on the posterior lower arch area, behind the second molar, plaque elementary lesion accomplished by reticular and also atrophic-erosive areas. The clinician who assisted the patient confirmed the same clinical diagnosis of the remote expert in all cases registered.

\section{CONClusions AND Future Works}

In this paper we presented a Teledentistry system aimed to Second Opinion. It is a low cost system allowing remote medical examinations when an oral medicine ward is not present in a medical structure or the opinion of a OME is necessary, because such a medical specialist is not present in the territory. The operator can perform a medical examinations session on the patients also when the OME is not logged to the account, even though the system allows to perform a real-time video streaming. A comparison between IOCs will be performed for future versions of this system. In this way, the system will be adaptive and will not be dependent from the specific IOC used in this prototype. We will try to develop a better integration of the IOC with the system. We will ask the collaboration with the research and development department of the IOC manufacturer.The medical quality of the real-time video streaming is sufficient to recognize heavy oral diseases, even though it is made in mepg 4 format, which performs a lossy compression. A dedicated line will be requested to the internet provider in order to obtain a larger bandwidth because we will investigate the possibility to perform a transmission using the H.264 format, without any compression. Moreover, the possibility to record the video streaming will be offered to the operator. In this way the OME will be able to analyze the video of a medical 


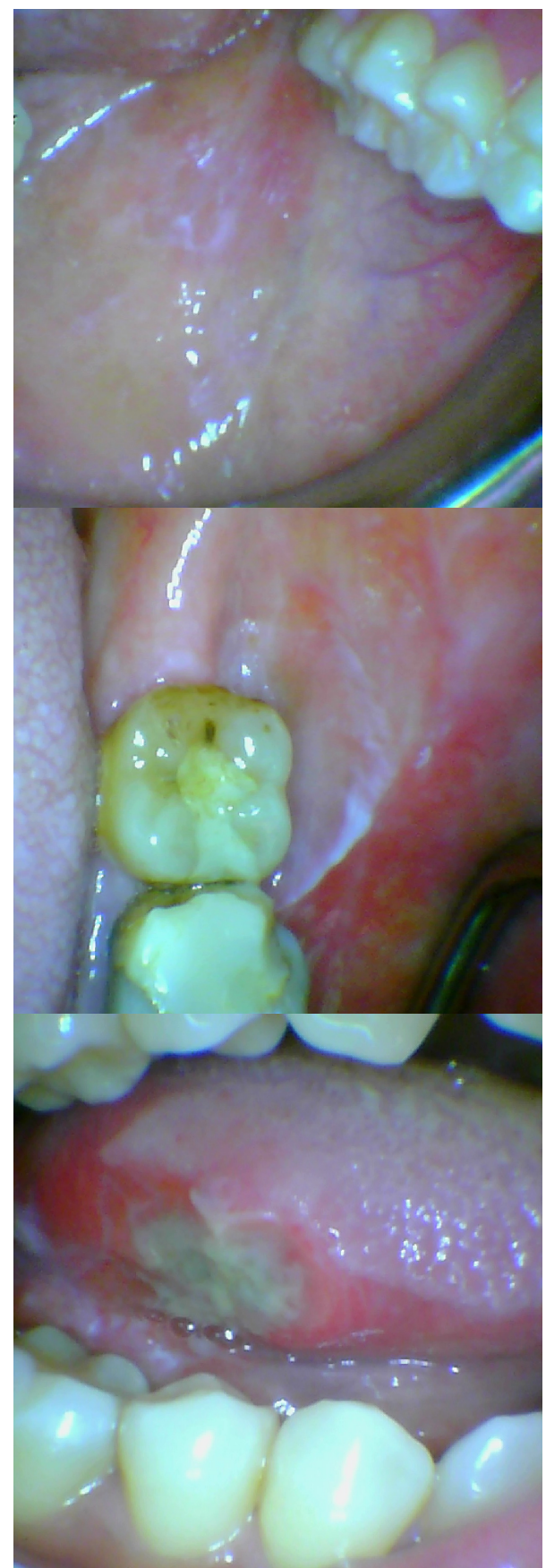

Fig. 4. Images acquired with the IOC HK 790 and transmitted to the OME.

examination like the other data. The system presented in this paper can be modified to be suited to other medical purposes, not just oral medicine. Indeed, many functionalities provided by the system are common to other telemedicine system In this way, in this way the system can be modified with limited efforts to achieve telemedicine systems for other medical specializations. Moreover, the acquisition equipment can be different from the one used in this work, which is directly connected to a USB port, but even though the new video source would be an analog signal, it can be easily converted using a video sampling device, which usually is a low cost device available in the market. In conclusion, the prototype presented in this paper is intended as the first step towards a larger Second Opinion platform to provide medical consulting service in different areas of medicine.

\section{ACKNOWLEDGMENT}

The authors express their gratefulness to the Rotary Club Palermo Teatro del Sole for the financial support given to this work.

\section{REFERENCES}

[1] Downer MC, Moles DR, Palmer S et al. A systematic review of test performance in screening for oral cancer and precancer. Oral Oncol 2004; 40: 264-73.

[2] Walsh T, Liu JL, Brocklehurst P, Glenny AM, Lingen M, Kerr AR, Ogden G, Warnakulasuriya S, Scully C. Clinical assessment to screen for the detection of oral cavity cancer and potentially malignant disorders in apparently healthy adults. Cochrane Database Syst Rev. 2013 Nov 21;11:CD010173. doi: 10.1002/14651858.CD010173.pub2.

[3] Bedogni A, Campisi G, Agrillo A, Fusco V; Expert Commission SICMF-SIPMO. Raccomandazioni clinico-terapeutiche sull'osteonecrosi delle ossa mascellari associata a bisfosfonati e sua prevenzione. Padova: CLEUP 2013.

[4] Bedogni A, Fusco V, Agrillo A, Campisi G. Learning from experience. Proposal for a refined definition and staging system for bisphosphonate-related osteonecrosis of the jaw (BRONJ). Oral Dis 2012;18(6):621-3.

[5] Ardizzone E, Gambino O, Genco A, Pirrone R, Sorce S (2009). Pervasive Access to MRI Bias Artifact Suppression Service on a Grid. Ieee Transactions On Information Technology In Biomedicine, vol. 13, p. 87-93, ISSN: 1089-7771, doi: 10.1109/TITB.2008.2007108

[6] Vandre, R.H. ; Kudryk, V.L. ; Fay, C.R., III ; Edwards, J.C., Jr. ; Jones, T.K. US Army teledentistry Proceedings of the National Forum Military Telemedicine On-Line Today, 1995. Research, Practice, and Opportunities.,DOI: 10.1109/MTOL.1995.504530 pp 53-56 (1995)

[7] Leao JC, Porter SR. Telediagnosis of oral disease Brazilian Dental Journal vol.10 n.1 pp: 1-60 ISSN 0103-6440 (1999)

[8] A.H. Forgie, C.M. Pine, N.B. Pitts The assessment of an intra-oral video camera as an aid to occlusal caries detection International Dental Journal Volume 53, Issue 1, pages 36, February 2003

[9] Chang SW, Plotkin DR, Mulligan R, Polido JC, Mah JK, Meara JG.Teledentistry in rural California: a USC initiative. Journal of the California Dental Association. Aug;31(8):601-8. 2003

[10] Sanchez Dils E, Lefebvre C, Abeyta K.Teledentistry in the United States: a new horizon of dental care. International Journal of Dental Hygiene. Nov;vol.2 n.4. pp 161-164. (2004)

[11] Xiao-Lin Lu System design and development for a CSCW based remote oral medical diagnosis system Proceedings of International Conference on Machine Learning and Cybernetics, 2005. pp: 3698-3703 vol 6

[12] R. N. Smith, A. Rawlinson, D. L. Lath, A.H. Brook. A digital SLR or intra-oral camera: preference for acquisition within an image analysis system for measurement of disclosed dental plaque area within clinical trials Journal of Periodontal Research Volume 41, Issue 1, pages 5561, February 2006

[13] Kopycka-Kedzierawski, DT and Billings, RJ.Teledentistry in innercity child-care centres Journal of Telemedicine Telecare. Vol.12, No.4,pp.176-81, ISSN 1357-633X. (2006)

[14] Berndt J, Leone P, King G. Using teledentistry to provide interceptive orthodontic services to disadvantaged children American Journal of Orthodontics and Dentofacial Orthopedics vol.34 n.5 pp. 700-706 (2008)

[15] Jian Yu Distributed Data Processing Framework for Oral Health Care Information Management Based on CSCWD Technology 1st International Conference on Information Science and Engineering (ICISE), 2009.Page(s): 2312 - 2315. DOI: 10.1109/ICISE.2009.511

[16] Zivkovic D., Tosic G., Mihailovic B., Miladinovic M., Vujicic B.Diagnosis of periapical lesions of the front teeth using the internet PONS Medical Journal, Volume 2010; vol.7 n.4- pp 138143 ISSN:1820-2411

[17] Brllmann D, Schmidtmann I, Warzecha K, and d'Hoedt B, Recognition of root canal orifices at a distance - a preliminary study of teledentistry Journal Of Telemedicine And Telecare, vol. 17, no. 3, pp. 154-157. (2011) 